\title{
A COHEN TYPE INEQUALITY FOR COMPACT LIE GROUPS
}

\author{
SAVERIO GIULINI, PAOLO M. SOARDI AND GIANCARLO TRAVAGLINI ${ }^{1}$
}

\begin{abstract}
The following theorem is proved: let $G$ denote a compact connected semisimple Lie group. There exists $\theta=\theta(G)(3<\theta<4)$ such that, if $\chi_{1}, \ldots, \chi_{N}$ are $N$ distinct characters of $G, d_{1}, \ldots, d_{N}$ their dimensions, $c_{1}, \ldots, c_{N}$ complex numbers of modulus greater than or equal to one, then, for all $p>\theta,\left\|\Sigma_{j-1}^{N} c_{j} d_{j} x_{j}\right\|_{p}>$ const $_{p} N^{a}$ where $\|\cdot\| \|_{p}$ denotes the $L^{P}(G)$ convolutor norm and const $p$ and $\alpha_{p}=\alpha_{p}(G)$ are positive constants. Results on divergence of Fourier series on compact Lie groups are deduced.
\end{abstract}

1. In a well-known paper [5] P. J. Cohen proved that if $\gamma_{1}, \ldots, \gamma_{N}$ are $N$ distinct characters of, say, the $n$-dimensional torus $\mathbf{T}^{n}$ and $c_{1}, \ldots, c_{N}$ are complex numbers of modulus larger than or equal to one, then the following inequality holds:

$$
\left\|\sum_{j=1}^{N} c_{j} \gamma_{j}\right\|_{L^{\prime}}>\operatorname{const}\left(\frac{\log N}{\log \log N}\right)^{1 / 8}
$$

(such an estimate was subsequently improved; see Pichorides [8]; [9]; see also [7]).

Let $T: L^{p} \rightarrow L^{p}$ denote the convolutor defined by $T f=\sum_{j=1}^{N} c_{j} \gamma_{j} * f, f \in$ $L^{p}$, and set

$$
\left\|\sum_{j=1}^{N} c_{j} \gamma_{j}\right\|_{p}=\operatorname{Sup}_{\|f\|_{L^{p}}=1}\|T f\|_{L^{p}}
$$

It is clear that in general it is impossible to obtain an inequality similar to (1) for $\left\|\mid \sum_{j=1}^{N} c_{j} \gamma_{j}\right\|_{p}$ if $p \neq 1(p \neq \infty)$, since it is known that square partial sums converge in $L^{p}\left(T^{n}\right)$.

Recently Clerc [4], Stanton [12] and Stanton and Tomas [13] studied the convergence of Fourier series in a compact Lie group. Clerc studied mean summability for spherical partial sums, while Stanton, and Stanton and Tomas, established several results for polyhedral partial sums, which resemble the square ones for the torus. However, unlike the abelian case, they proved that if $p \neq 2$ there is $f \in L^{p}$ whose polyhedral partial sums do not converge in $L^{p}$ norm. The object of this paper is to prove a result which may help to clarify this difference. Namely, we prove a Cohen type inequality for the $L^{p}(G)$ convolutor norm of $\sum_{j=1}^{N} c_{j} d_{j} \chi_{j}$, where $\chi_{1}, \ldots, \chi_{N}$ are $N$ distinct

Received by the editors December 11, 1978.

AMS (MOS) subject classifications (1970). Primary 43A55, 43A50; Secondary 22E30.

Key words and phrases. Compact Lie groups, Dirichlet kernels, convolutors.

${ }^{1}$ This work was partially supported by the C. N. R. 
characters of $G, d_{j}$ their dimensions, $c_{1}, \ldots, c_{N}$ complex numbers such that $\left|c_{j}\right|>1, j=1, \ldots, N$, and $p$ is larger than an index $\theta$ depending only on $G$ (or smaller than the conjugate index $\theta^{\prime}$ ). As a consequence we get some results about the $L^{p}$ norm divergence of partial sums of Fourier series, taken with respect to any way of grouping the elements of the dual object of $G$. When $p=1$ we also get an analogue of (1) for compact connected semisimple Lie groups which answers a question implicitly raised in [10] and [11].

Before stating explicitly our results, we introduce the notation that will be used throughout the paper.

Let $G$ denote a $n$-dimensional compact connected semisimple Lie group with Lie algebra $\mathscr{L}(G)$ and let $T$ denote a maximal torus of $G$ with Lie algebra $\mathcal{L}(T)$. The complexification $\mathscr{L}_{C}(T)$ is a Cartan subalgebra of $\mathscr{L}_{C}(G)$, and we will denote by $\Delta$ the set of roots of $\left(\mathscr{L}_{c}(G), \mathscr{L}_{C}(T)\right)$. We choose in $\Delta$ a system of positive roots $P$ and denote by $S=\left(\alpha_{1}, \ldots, \alpha_{r}\right)$ the associated system of simple roots. The irreducible unitary representations of $G$ are in one-to-one correspondence with the semilattice $\Sigma$ of the dominant weights of $G$. The weights of $G$ are usually ordered by letting $\lambda_{1} \leqslant \lambda_{2}$ if $\lambda_{2}-\lambda_{1}$ is a sum (possibly empty) of simple roots (see e.g. [15, p. 314]). We write $\lambda_{1} \prec \lambda_{2}$ if $\lambda_{1} \preccurlyeq \lambda_{2}, \lambda_{1} \neq \lambda_{2}$. For every $\omega \in \Sigma$ we shall denote by $\chi_{\omega}$ and $d_{\omega}$ the character and the dimension respectively of the corresponding representation of $G$. Finally, for a central function $f \in L^{1}$, we denote by $\|f\|_{p}(1<p<\infty)$ the norm of the operator $T_{f}: L^{p} \rightarrow L^{p}$ defined by $T_{f} g=f * g, g \in L^{p}$. Clearly, if $p$ and $p^{\prime}$ are conjugate exponents, $\|f\|_{p}=\|\| f \|_{p^{\prime}}$.

TheOREM. Let $G$ denote a compact connected semisimple Lie group. There exists $\theta=\theta(G)(3 \leqslant \theta<4)$ such that, if $\chi_{1}, \ldots, \chi_{N}$ are $N$ distinct characters of $G, c_{1}, \ldots, c_{N}$ complex numbers of modulus greater than or equal to one, then, for all $p>\theta$,

$$
\left\|\left|\sum_{j=1}^{N} c_{j} d_{j} x_{j}\right|\right\|_{p}>\text { const }_{p} N^{\alpha_{p}}
$$

where const $t_{p}$ and $\alpha_{p}=\alpha_{p}(G)$ are positive constants.

It should be remarked that our result depends heavily on A. H. Dooley's estimates for norms of characters of $G$ [6] (see also Cecchini [3]).

The proof of this theorem will be given in the next section. An expression for the constants $\theta$ and $\alpha_{p}$ is given in equations (11)-(14).

2. Suppose $\sigma_{1}$ and $\sigma_{2}$ are dominant weights of $G$ such that $\sigma_{1} \leqslant \sigma_{2}$. It is not true in general that $d_{\sigma_{1}} \leqslant d_{\sigma_{2}}$; however we have the following bound for $d_{\sigma}$.

Lemma. Suppose that $\sigma_{1}$ and $\sigma_{2}$ are dominant weights of $G, \sigma_{1} \leqslant \sigma_{2}$. Then $d_{\sigma_{1}}<$ const $d_{\sigma_{2}}^{q}$, where $q=$ card $P$. 
Proof. Let $\Omega_{1}, \ldots, \Omega_{r}$ denote the fundamental weights of the Lie algebra $\varrho_{C}(T)$. Then, if $\beta=\frac{1}{2} \Sigma_{\alpha \in P} \alpha, \sigma_{i}+\beta=\sum_{j=1}^{r} m_{i, j} \Omega_{j}(i=1,2)$ where the $m_{i, j}$ are positive integers. We denote by $\mathscr{L}_{\mathrm{C}}(T)^{*}$ the dual space of $\mathcal{L}_{\mathrm{C}}(T)$ and transfer the Killing form to a symmetric nonsingular bilinear form $\langle\cdot, \cdot\rangle$ on $\mathfrak{L}_{\mathbf{C}}(T)^{*} \times \mathfrak{L}_{\mathbf{C}}(T)^{*}$ via the natural isomorphism of $\mathfrak{L}_{\mathbf{C}}(T)$ with $\mathfrak{E}_{\mathbf{C}}(T)^{*}$. Hence we have, for every $j=1, \ldots, r$,

$$
m_{1, j}<\operatorname{const}\left\langle\sigma_{1}+\beta, \Omega_{j}\right\rangle\left\langle\operatorname{const}\left\langle\sigma_{2}+\beta, \Omega_{j}\right\rangle\right.
$$

since $\sigma_{1} \preccurlyeq \sigma_{2}$. Moreover

$$
\left\langle\sigma_{2}+\beta, \Omega_{j}\right\rangle=\sum_{h=1}^{r} m_{2, h}\left\langle\Omega_{h}, \Omega_{j}\right\rangle\left\langle\text { const } \prod_{h=1}^{r} m_{2, h} .\right.
$$

By Weyl's dimension formula:

$$
d_{\sigma_{2}}>\text { const } \prod_{h=1}^{r} m_{2, h} \cdot \prod_{\alpha \in P \backslash S}\left\langle\sigma_{2}+\beta, \alpha\right\rangle>\text { const } \prod_{h=1}^{r} m_{2, h}
$$

since

$$
\left.\prod_{\alpha \in P \backslash S}\left\langle\sigma_{2}+\beta, \alpha\right\rangle\right\rangle \prod_{\alpha \in P \backslash S}\langle\beta, \alpha\rangle .
$$

Hence, by Weyl's dimension formula again, (3) and (4),

$$
\begin{aligned}
d_{\sigma_{1}} & =\frac{\Pi_{\alpha \in P} \sum_{j=1}^{r} m_{1, j}\left\langle\Omega_{j}, \alpha\right\rangle}{\prod_{\alpha \in P}\langle\beta, \alpha\rangle} \\
& \left\langle\text { const } \prod _ { \alpha \in P } d _ { \sigma _ { 2 } } \sum _ { j = 1 } ^ { r ^ { \cdot } } \langle \Omega _ { j } , \alpha \rangle \left\langle\text { const } d_{\sigma_{2}}^{q} .\right.\right.
\end{aligned}
$$

For every weight $\lambda$ of $G$ let $\xi_{\lambda}: T \rightarrow C$ denote the character of $T$ which makes the following diagram commutative:

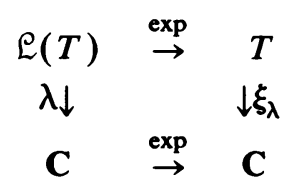

We recall that $\lambda$ takes only pure imaginary values on $\mathcal{L}(T)$ and the correspondence between $\lambda$ and $\xi_{\lambda}$ is one-to-one.

The symmetric sums $S(\lambda): T \rightarrow C$ are defined in the usual way: $S(\lambda)$ : $t \rightarrow \Sigma_{\mu} \xi_{\mu}(t)$ where the summation is over all $\mu$ in the orbit of $\lambda$ under the action of the Weyl group $W . \tilde{S}(\lambda)$ will denote the unique central continuous extension of $S(\lambda)$ to the whole of $G$. We define also the alternating sums $A(\lambda)$ : $T \rightarrow \mathrm{C}, A(\lambda): t \rightarrow \Sigma_{\phi \in W} \tau_{\phi} \xi_{\phi(\lambda)}(t)$ where $\tau_{\phi}$ is the determinant of $\phi$.

Suppose that $\mathscr{L}_{\mathbf{C}}(T)$ factors through the simple Lie algebras $\mathscr{L}_{i}, \mathscr{L}_{\mathbf{C}}(t)=$ $\bigoplus_{j=1}^{k} \mathcal{L}_{i}$. Let $r_{i}$ and $q_{i}$ denote the rank and the number of positive roots of $\mathscr{L}_{i}$, respectively. We let

$$
c=\max _{i=1, \ldots, k} r_{i} / q_{i}
$$


and let $s$ denote the number of algebras $\mathcal{L}_{i}$ for which the ratio $r_{i} / q_{i}$ is equal to c. We now are ready to prove the theorem.

Proof of THe Theorem. First suppose that $\beta=\frac{1}{2} \Sigma_{\alpha \in P} \alpha$ is a weight of $G$. Let $\delta=A(\beta)$ and let $\omega$ denote any dominant weight of $G$. We want to show that $\chi_{\omega}$ is the lowest term in the Fourier expansion of $\tilde{S}(\omega+2 \beta)$. This can be achieved by a variant of a known argument. We have

$$
\begin{aligned}
S(\omega+2 \beta) \delta & =\sum_{\phi, \psi \in W} \tau_{\psi} \xi_{\phi(\omega+2 \beta)+\psi(\beta)} \\
& =\sum_{\psi \in W} \tau_{\psi} \sum_{\phi \in W} \tau_{\phi} \xi_{\phi(\omega+2 \beta+\psi(\beta))}=\sum_{\psi \in W} \tau_{\psi} A(\omega+2 \beta+\psi(\beta))
\end{aligned}
$$

where $A(\lambda)=0$ whenever $\lambda$ is singular. Hence we can write

$$
S(\omega+2 \beta) \delta=\sum_{\psi \in W} \tau_{\psi} \tau_{\tilde{\phi}} A(\tilde{\phi}(\omega+2 \beta+\psi(\beta)))
$$

where $\tilde{\phi}(\omega+2 \beta+\psi(\beta))$ is the unique nonsingular dominant weight in the orbit of $\omega+2 \beta+\psi(\beta)$. Formula (6) can also be written

$$
S(\omega+2 \beta) \delta=\sum n_{j} A\left(\omega_{j}+\beta\right)
$$

where the $\omega_{j}$ are distinct dominant weights and the $n_{j}$ are relative integers. Let $\iota \in W$ denote the identity and $-\imath$ the element such that $-\imath P=-P$. It is clear that if in (6) $\psi=\imath$, then $\tilde{\phi}=\imath$ and we get the sum $A(\omega+3 \beta)$. If $\psi=-\iota$ then $\tilde{\phi}=\imath$ and we get the sum $A(\omega+\beta)$. Hence (7) becomes

$$
S(\omega+2 \beta) \delta=A(\omega+3 \beta)+\sum n_{j} A\left(\omega_{j}+\beta\right)+\tau A(\omega+\beta)
$$

where $\tau$ is equal to +1 or -1 and $\omega_{j} \neq \omega+2 \beta, \omega_{j} \neq \omega$. For such an $\omega_{j}$ we have

$$
\omega \prec \omega_{j} \prec \omega+2 \beta
$$

since $\tilde{\phi}(\omega+2 \beta+\psi(\beta))<\omega+3 \beta$ and $\tilde{\phi}(\omega+2 \beta+\psi(\beta))>\omega+2 \beta+$ $\psi(\beta)>\omega+\beta$ (since $\tilde{\phi}(\omega+2 \beta+\psi(\beta))$ is dominant, (see [15, 4.7.3])). Hence, by Weyl's character formula

$$
\tilde{S}(\omega+2 \beta)=\chi_{\omega+2 \beta}+\sum n_{j} \chi_{\omega j}+\tau \chi_{\omega}
$$

If $\beta$ is not a weight of $G$ it is possible to prove (10), with the $\omega_{j}$ satisfying (9), by passing to the double covering of $G$ and then arguing as in $[1,6.33]$.

Suppose now that we are given $N$ distinct characters $\chi_{\omega_{1}}, \ldots, \chi_{\omega_{N}}$ and $N$ complex numbers $c_{1}, \ldots, c_{N},\left|c_{j}\right|>1, j=1, \ldots, N$. Assume that $d_{\omega_{N}}>d_{\omega}$ for all $j, 1<j<N$. There exists $\rho \in\left\{\omega_{1}, \ldots, \omega_{N}\right\}$ such that $\omega_{N} \leqslant \rho$ and, for every $\omega \in\left\{\omega_{1}, \ldots, \omega_{N}\right\}$, either $\omega$ is not comparable with $\rho$ or $\omega \preccurlyeq \rho$. We have

$$
\|\tilde{S}(\rho+2 \beta)\|_{L^{p}}<\|\tilde{S}(\rho+2 \beta)\|_{\infty}=|W|
$$


Therefore, by (9) and (10),

$$
\begin{aligned}
\|\| \sum_{j=1}^{N} c_{j} d_{\omega j} \chi_{\omega j}\|\|_{p} & \geqslant|W|^{-1}\left\|\sum_{j=1}^{N} c_{j} d_{\omega,} \chi_{\omega,} * \tilde{S}(\rho+2 \beta)\right\|_{L^{p}} \\
& =|W|^{-1}\left\|\chi_{\rho}\right\|_{L^{p}}
\end{aligned}
$$

for all $p \in[1, \infty]$.

Let

$$
\theta=4-\min _{i=1, \ldots, k} q_{i}^{-1}
$$

Combining the assertions (ii) and (iii) of Theorem (5.4) in Dooley's paper [6] we conclude that there is a positive constant $\lambda_{p}$ such that, for all $p>\theta$,

$$
\left\|x_{\rho}\right\|_{L^{p}} \geqslant \text { const }_{p} d_{\rho}^{\lambda_{p}} \text {. }
$$

For large values of $p$

$$
\lambda_{p}=1-M_{G} / p
$$

where $M_{G}$ depends only on $G$, while, for $p<4$,

$$
\lambda_{p}=1-\theta / p
$$

Hence, by the previous lemma,

$$
\left\|\mid \sum_{j=1}^{N} c_{j} d_{\omega_{j}} \chi_{\omega_{j}}\right\| \|_{p}>d_{\omega_{N}}^{q^{-1} \lambda_{j}} .
$$

It is now a consequence of a theorem of R. S. Cahn [2] that $d_{\omega_{N}}^{q^{-1}}>$ const $N^{\gamma}$ provided that

$$
\gamma=(c+s-1)^{-1} q^{-1}
$$

so that (2) follows.

3. We end the paper by drawing some consequences of the theorem just proved. Throughout this section, $G$ denotes a compact connected semisimple Lie group.

Suppose $\left\{\Sigma_{N}\right\}$ is an increasing sequence of finite subsets of $\Sigma$ such that $\cup_{n=1}^{\infty} \Sigma_{N}=\Sigma$. For every integrable $f$ we define the partial sums relative to $\left\{\Sigma_{N}\right\}$ by

$$
S_{N} f=\sum_{\omega \in \Sigma_{N}} d_{\omega} \chi_{\omega} * f
$$

Clearly each choice of the sequence $\left\{\Sigma_{N}\right\}$ gives rise to a different way of interpreting the convergence of Fourier series.

Since the test functions $\tilde{S}(\omega+2 \beta)$ used to establish (2) are central we have the following corollary.

Corollary 1. For every $p, 1<p<\theta^{\prime}$, or $p>\theta$, and for every choice of the sequence $\left\{\Sigma_{N}\right\}$ there exists a central $f \in L^{p}(G)$ such that

$$
\underset{N \rightarrow \infty}{\lim \sup }\left\|S_{N} f\right\|_{L^{p}}=\infty \text {. }
$$


We notice that, for simple groups $G$ and polyhedral partial sums $S_{N} f$, Stanton and Tomas' result is sharper than ours, since $\theta \geqslant 2 n /(n-r)$.

Corollary 2. For every $p, 1<p<\theta^{\prime}$, and every choice of the sequence $\left\{\Sigma_{N}\right\}$ there exists a function $f \in L^{p}(G)$ such that

$$
\limsup _{N \rightarrow \infty}\left|S_{N} f(x)\right|=\infty
$$

for almost all $x \in G$.

This is a consequence of Corollary 1 and a result of Stein [14].

We note also that, for $p=1$, we have an estimate similar to (1).

COROLlaRY 3. If $\chi_{\omega_{1}}, \ldots, \chi_{\omega_{N}}$ are $N$ distinct characters and $c_{1}, \ldots, c_{N}$ are complex numbers such that $\left|c_{j}\right|>1, j=1, \ldots, N$, then

$$
\left\|\sum_{j=1}^{N} c_{j} d_{\omega_{j}} \chi_{\omega_{j}}\right\|_{L^{1}}>\text { const } N^{\gamma} \text {. }
$$

\section{REFERENCES}

1. J. F. Adams, Lectures on Lie groups, Benjamin, New York, 1969.

2. R. S. Cahn, Lattice points and Lie groups. I, Trans. Amer. Math. Soc. 183 (1973), 119-129.

3. C. Cecchini, Lacunary Fourier series on compact Lie groups, J. Functional Analysis 11 (1972), 191-203.

4. J. L. Clerc, Sommes de Riesz and multiplicateurs sur un groupe de Lie compact, Ann. Inst. Fourier 24 (1974), 149-172.

5. P. J. Cohen, On a conjecture of Littlewood and idempotent measures, Amer. J. Math. 82 (1960), 191-212.

6. A. H. Dooley, Norms of characters and lacunarity for compact Lie groups, J. Functional Analysis (to appear).

7. E. Hewitt and H. S. Zuckerman, On a theorem of P.J. Cohen and H. Davenport, Proc. Amer. Math. Soc. 14 (1963), 847-855.

8. S. K. Pichorides, $A$ lower bound for the $L^{1}$ norm of exponential sums, Matematika 21 (1974), $155-159$.

9. , On a conjecture of Littlewood concerning exponential sums, Notices Amer. Math. Soc. 24 (1977), A-427.

10. J. F. Price, On the integral divergence of Dirichlet kernels for the second unitary and special unitary groups, J. London Math. Soc. (2) 9 (1975), 593-598.

11. On local central lacunary sets for compact Lie groups, Monatsh. Math. 80 (1975), no. 3, 201-204.

12. R. J. Stanton, On mean convergence of Fourier series on compact Lie groups, Trans. Amer. Math. Soc. 218 (1976), 61-87.

13. R. J. Stanton and P. A. Tomas, Polyhedral summability of Fourier series on compact Lie groups, Amer. J. Math. 100 (1978), 477-493.

14. E. M. Stein, On limits of sequences of operators, Ann. of Math. 74 (1961), 140-170.

15. V. S. Varadarajan, Lie groups, Lie algebras and their representations, Prentice-Hall, Englewood Cliffs, N.J., 1974.

Istituto Matematico Dell' Universita, Via Saldini 50, 20133 Milano, Italy 